\title{
Characteristics of Patients with Tramadol Use or Abuse: A Systematic Review and Meta-Analysis
}

\author{
(1) Paria Habibollahi ${ }^{1}$, (1) Alireza Garjani², (1) Samad Shams Vahdati ${ }^{3}$, (1) Seyyed-Reza Saadat Ebrahimi ${ }^{4}$, (1) Neda Parnianfard ${ }^{5}$, \\ (D) Raana Zakeri6 \\ 1Pharmaceutical Analysis Research Center, Tabriz University of Medical Sciences, Tabriz, Iran \\ 2Department of Pharmacology and Toxicology, Tabriz University of Medical Sciences, Tabriz, Iran \\ ${ }^{3}$ Emergency Medicine Research Team, Tabriz University of Medical Sciences, Tabriz, Iran \\ ${ }^{4}$ Research Center for Evidence-Based Medicine, Iranian Evidence-Based Medicine Center: A Joanna Briggs Institute Affiliated Group, Health Management \\ and Safety Promotion Research Institute, Tabriz University of Medical Sciences, Tabriz, Iran \\ ${ }^{5}$ Drug Applied Research Center, Tabriz University of Medical Sciences, Tabriz, Iran \\ ${ }^{6}$ Department of Health Services Management, School of Management and Medical Informatics, Iranian International Safe Community Support Center, \\ Tabriz University of Medical Sciences, Tabriz, Iran
}

\begin{abstract}
Recently, an increasing number of studies have reported an association between the incidence of seizures and the use or abuse of tramadol. The aim of this study was to review articles involving patients with tramadol-induced seizures that reported their characteristics and the consumed tramadol dosage.

The following databases were searched from their inception until October 30, 2018: PubMed, EMBASE, the Cochrane Library, Scopus, Ovid, Proquest and Science direct. All cross-sectional studies that reported the tramadol dosage and individual characteristics of patients with tramadol-induced seizure were included. The studies were assessed for any bias risk using the adapted Newcastle-Ottawa scale. The pooled estimates were reported using random-effects meta-analysis.

Our search identified a total of 3,275 articles, and we finally extracted data from 11 studies involving 970 patients. The included studies were all conducted in developing countries. The pooled data resulted in an estimated mean ( \pm standard deviation) of 1,454.5 $\pm 333.6 \mathrm{mg}$ for tramadol dosage that induces seizures in patients. The mean minimum tramadol dose causing seizure in the pooled data was $169.5 \pm 131.2$ $\mathrm{mg}$. The mean age of the patients was $25.85 \pm 0.86$ years. In addition, $83.37 \pm 12.6 \%$ of the patients were male, and the manner of poisoning in $70.51 \pm 29.07 \%$ of the patients was suicide or abuse.

Our findings demonstrated that the prevalence of seizures following the consumption of tramadol was higher among males. Therefore, the probability of seizure should be considered by physicians in prescribing tramadol, and the individuals receiving it, especially the ones at a higher risk of exposure, should be properly informed about its complications.

Keywords: Tramadol, overdose, seizure, systematic review, meta-analysis
\end{abstract}

\section{Introduction}

Tramadol is an atypical synthetic opioid analgesic which is administered as a potent treatment for acute (such as postoperative or trauma) and chronic (such as cancer) pain since 1977. Moreover, it has some reinforcing/rewarding effects which are caused by activation of $\mu$-opioid and monoamine receptor systems $(1,2)$.
Because of having similar effects on other opioid receptor agonists, the risk of tramadol abuse exists in certain populations (3) (ambiguous). In 2013, The International Narcotics Control Board ran a survey in 32 countries, and estimated the tramadol abuse accounts as 69 in 1000 person per year (4). The indicated therapeutic range for moderate to severe pain is 25 up to $400 \mathrm{mg} /$ day in form of oral dosage (disintegrating tablets). Also, for chronic

Cite this article as: Habibollahi P, Garjani A, Vahdati SS, Ebrahimi SRS, Parnianfard N, Zakeri R. Characteristics of Patients with Tramadol Use or Abuse: A Systematic Review and Meta-Analysis. Eurasian J Emerg Med. 2020;19(3):127-35

(C) Copyright 2020 by the Emergency Medicine Physicians' Association of Turkey

Eurasian Journal of Emergency Medicine published by Galenos Publishing House. 
pain, the advised oral dosage form (extended-release capsules) is 100 up to $300 \mathrm{mg} /$ day. Tramadol overdose can bring about acute renal failure, rise in creatinine phosphokinase, hepatic failure, electrocardiographic changes and acute right heart dysfunction $(5,6)$. Also, there are few death reports caused by tramadol abuse $(7)$.

Although a growing number of recent studies has been reported an association between the incidence of seizures and using/ abusing tramadol (8-10), still the mechanism of tramadol in the incidence of seizures is poorly understood; It has been assumed that high concentrations of tramadol inhibiting gammaaminobutyric acid receptors are associated with the incidence of seizures in animal models (11). In addition, the characteristics of patients with tramadol induced seizure and the consumed tramadol dosage in them varies greatly among different studies (12-21). Since no previous review study has intended to collect this discrete data and put forward reliable and coherent information of tramadol to help physicians and patients to use it safely, the current study aimed to review the articles over the patients with tramadol induced seizure which reported their characteristics and consumed tramadol dosage.

\section{Study Method}

A systematic review and meta-analysis of cross-sectional studies over tramadol induced seizure was conducted.

\section{Search Strategy}

A skilled librarian defined the search strategies for some of the most important bibliographic databases including PubMed, EMBASE, the Cochrane Library, Scopus, Ovid, Proquest, and Science direct until 30 October 2018. All search data were imported to reference manager software, EndNote X8. The keywords for the search strategy for PubMed were as follows: Seizures AND Tramadol/(Seizure or Convulsion or Convulsive) and (Tramadol)/(Seizure or Convulsion or Convulsive) and (Tramadol or Tramundin or Biodalgic or Jutadol or K-315 or K 315 OR K315 or MTW-Tramadol or MTW Tramadol or MTWTramadol or Nobligan or ProntofORt or Zytram or Takadol or Theradol or Tiral or Tramadol Lindo or Topalgic or Tradol or Tradol-Puren or Tradol Puren or TradolPuren or Tradonal or Tralgiol or Trama AbZ or Trama KD or Trama-DORsch or Trama DORsch or TramaDORsch or Biokanol or Tramabeta or Tramadin or Tramadoc or Ranitidin 1A Pharma or Trama 1A Pharma or Trasedal or Ultram or Xymel 50 or Zamudol or Zumalgic or Zydol or TramadolHameln or TramadolOR or Tramadura or Tramagetic or Tramagit or Tramake or Tramal or Tramex or Adolonta or Contramal or Amadol).

\section{Study Selection}

At the first screening stage, two reviewers independently screened title and abstract of retrieved documents in order to determine the ones which met the eligibility criteria. Primary selection of studies was based on the inclusion criteria. The duplicated publications were excluded. Full citations of documents that were considered eligible at least by one reviewer were imported into an EndNote database. In the next stage, the full text of the imported papers was provided and reviewed for subject relevancy by the two reviewers individually. A critical appraisal checklist was used to evaluate the validity of the selected studies and to criticize them. Finally, these two researchers made a faceto-face meeting, discussing article selections. If they did not come to a consensus in a case, a third researcher made the final decision on the eligibility of that particular article. Consequently, the studies that considered valid by both researchers, selected for data extraction.

We included the cross-sectional studies (including the studies with retrospective or prospective observational designs) reporting the patients' characteristics and tramadol dosage in adult patients who received tramadol with or without a prescription and admitted with tramadol induced seizure. The full text of studies in English language were reviewed. The following studies were excluded: laboratory or animal studies, studies published only as synopsis or abstract, studies reporting the use/abuse of tramadol in combination with other co-ingestants or having seizure-related comorbidities, the studies which did not specifically reported the patients' data, studies on efficacy or safety of tramadol (e.g. drug half-life), studies on other adverse effects of tramadol, short communications and case reports. (Find the PRISMA checklist in S1).

\section{Risk of Bias Assessment}

The quality of studies was evaluated individually by two authors using the Newcastle-Ottawa scale adapted for cross-sectional studies (22), which evaluates the studies in three domains of selection, comparability, and outcome. Each one of the studies received a score ranging from 0 to 11 based on the sum of scores they gained in each domain (The details of quality assessment is available in our electronic supplementary material, S2). Other co-authors were consulted in case of no agreement between the two authors.

\section{Data Extraction}

The extraction table of our review included the following variables: characteristics of the patients (age and gender), consumed tramadol dosage, history of tramadol abuse, route of exposure, manner of poisoning, previous drug or substance abuse, history of any attempts for suicide, type of the seizure, number of episodes of seizure were extracted from the included studies (ambiguous). Two reviewers independently abstracted 
the mean and standard deviation (SD) of the review variables from their assigned study and extracted them into the extraction table. For the studies, which were the review variables as mean amounts without SDs, the $\mathrm{p}$ values were extracted. In addition, the sample size of each included study was added to the extraction table.

\section{Statistical Analysis}

The meta-analysis was utilized for combination and calculation of the review variables. The heterogeneities of review variables along the included study were assessed using Cochran's $Q$ and I2 statistic. Negative values of 12 are put equal to $0 \%$ so 12 values can range between $0 \%$ and $100 \%$. Zero percent indicates no observed heterogeneity; whereas larger values indicate increasing heterogeneity. Subgroup analyses were used to explore the reasons for heterogeneity. For the variables with a high degree of heterogeneity, the Der-Simonian-Laird method for a random-effect model was used to estimate the overall effect, and for the variables with a small degree of heterogeneity, the Mantel-Haenszel fixed-effect model was used. All statistical analyses were performed using CMA v.2.0 software.

\section{Findings}

\section{Literature Search}

Our comprehensive search strategy resulted in 3,275 preliminary articles that were screened by title and abstract. Lastly, full papers of 30 studies were evaluated for eligibility, of which 19 studies were excluded (studies on the combination of tramadol with other drugs $(n=3)$, discussing other adverse effects of tramadol $(n=4)$, Short communication $(n=3)$, and not specifically related to our scope $(n=9))$ and the full texts of 11 studies were critically reviewed (Figure 1).

\section{Bias Assessment}

After full text screening, risk of bias was assessed for remaining studies (Table 1). The agreement score between two authors in risk of bias assessment was 89 percent; discrepancies were resolved by discussion and consulting other authors. Most of the studies were of high quality and provided appropriate details about their outcomes and comparability; however, few trials presented clear complete details of their selection procedure and adequate information regarding ascertainment of true seizure and ascertainment of the exposure to tramadol. Also, no significant publication bias was demonstrated in our analysis [Kendall's tau with continuity correction $=-0.035, \mathrm{p}$ value (two tailed) $=0.9015]$.

\section{Tramadol Dosage in Patients with Tramadol Induced Seizure}

Finally, 11 studies were included. The characteristics of included studies are presented in (Tables 2, 3). The studies were mostly conducted in Iran (nine studies) and Egypt (two studies).

Of 11 included studies, eight studies reported the mean and SD of ingested tramadol dosage inducing seizure; however, two studies reported median and range $(17,19)$ and one study only reported the range of ingested tramadol dosage inducing seizure (14). Therefore, the results of those eight studies were used to calculate estimated mean dose. The pooled data of eight studies with 760 patients resulted in an estimated mean of 1,454.5 \pm 333.6 $\mathrm{mg}$ for tramadol dosage inducing seizure in patients (Figure 2). The heterogeneity between the included study was significant $(Q=6160.68,12=99.88, p<0.001)$. The average of each of the minimum values for tramadol dosage in patients with tramadol induced seizure were reported in 10 studies with 874 patients and one study did not report the minimum dose (13). The estimation over the minimum values of consumed tramadol dosage yield a

Table 1. Results of the assessments of the included studies by Newcastle-Ottawa Checklist* adopted for cross-sectional studies

\begin{tabular}{|l|l|l|l|l|l|}
\hline Author & Year & $\begin{array}{l}\text { Selection } \\
\text { (maximum 6 stars) }\end{array}$ & $\begin{array}{l}\text { Comparability } \\
\text { (maximum 2 stars) }\end{array}$ & $\begin{array}{l}\text { Outcome } \\
\text { (maximum 3 stars) }\end{array}$ & $\begin{array}{l}\text { Quality points total } \\
\text { (maximum 11 stars) }\end{array}$ \\
\hline Petramfar et al. (20) & 2009 & 3 & 1 & 3 & 7 \\
\hline Talaie et al. (15) & 2009 & 3 & 2 & 3 & 8 \\
\hline Goodarzi et al. (21) & 2011 & 2 & 2 & 3 & 7 \\
\hline Taghaddosinejad et al. (11) & 2011 & 4 & 2 & 3 & 9 \\
\hline Farajidana et al. (9) & 2012 & 4 & 2 & 3 & 9 \\
\hline Rahimi et al. (16) & 2014 & 2 & 2 & 3 & 7 \\
\hline Enaba et al. (17) & 2015 & 4 & 2 & 3 & 9 \\
\hline Asadi et al. (18) & 2015 & 3 & 2 & 3 & 8 \\
\hline Rizk et al. (13) & 2016 & 6 & 2 & 3 & 11 \\
\hline Ahmadimanesh et al. (19) & 2018 & 3 & 2 & 3 & 8 \\
\hline Mohammadpour et al. (14) & 2018 & 4 & 2 & 9 \\
\hline *Cross-sectional studies: very good studies: 9-11 points, good studies: 7-8 points, satisfactory studies: 5-6 points, unsatisfactory studies: 0 to 4 points \\
\hline
\end{tabular}




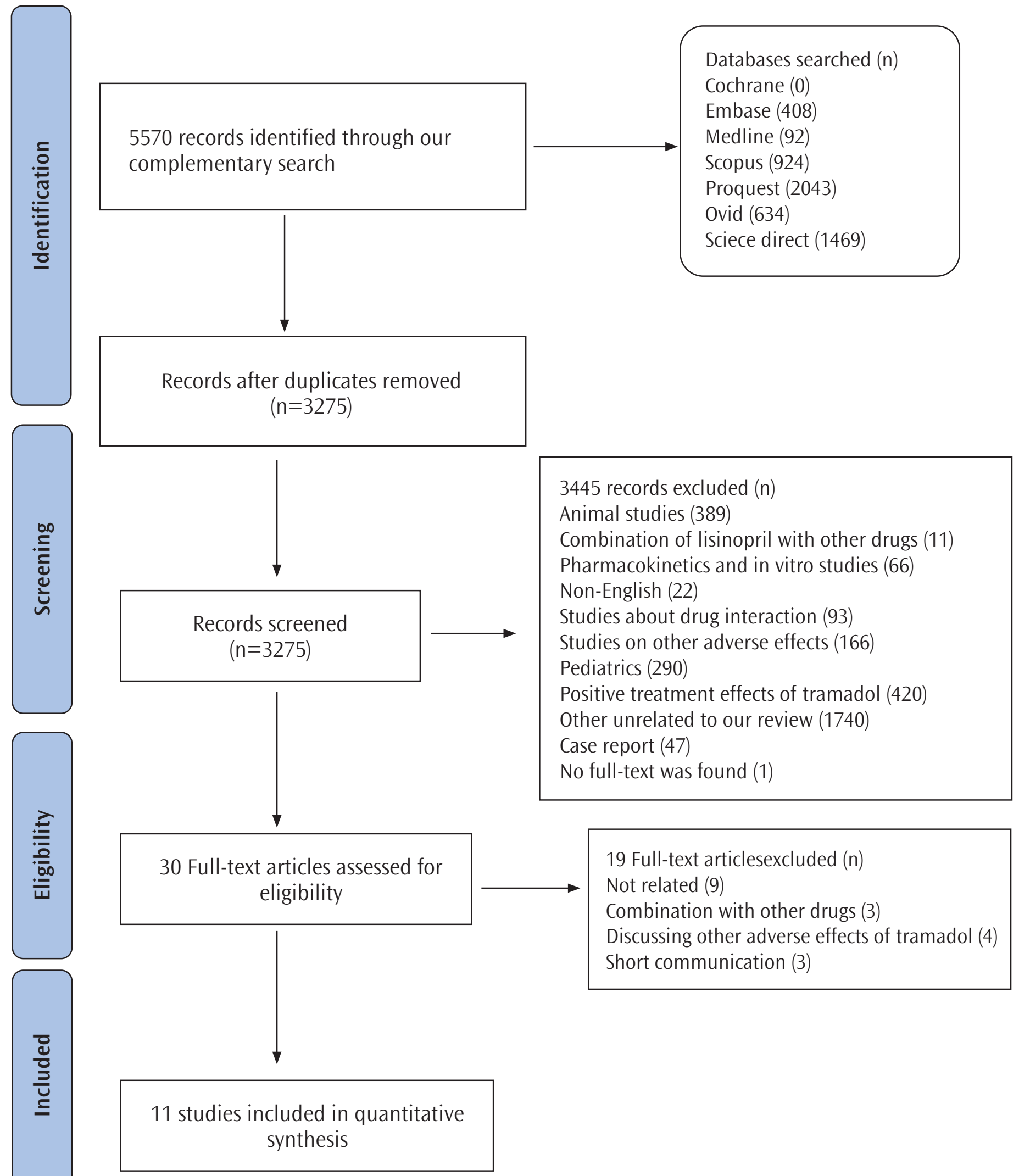

Figure 1. PRISMA flow diagram summarizing retrieved, included, and excluded studies n: Number, PRISMA: Preferred Reporting Items for Systematic Reviews and Meta-analyses 


\begin{tabular}{|c|c|c|c|c|c|c|}
\hline Author & Year & Study design & Country & $\begin{array}{l}\text { Mean age } \\
\text { (range) }\end{array}$ & $\begin{array}{l}\text { Sex male in seizure } \\
\text { group number }\end{array}$ & $\begin{array}{l}\text { Sex female in } \\
\text { seizure group }\end{array}$ \\
\hline Petramfar et al. (20) & 2009 & Cross-sectional & Iran & $26.7 \pm 6.9$ & 102 & 4 \\
\hline Talaie et al. (15) & 2009 & Cross-sectional & Iran & $24.3(15-69)$ & 51 & 10 \\
\hline Goodarzi et al. (21) & 2011 & Cross-sectional & Iran & $26.48 \pm 7.74(17-45)$ & 27 & 27 \\
\hline Taghaddosinejad et al. (11) & 2011 & Observational prospective & Iran & $22.9(14-50)$ & 101 & 20 \\
\hline Farajidana et al. (9) & 2012 & Retrospective & Iran & $23 \pm 6(60-3)$ & 197 & 35 \\
\hline Rahimi et al. (16) & 2014 & Retrospective & Iran & $24.2 \pm 7.6(16-57)$ & 57 & 12 \\
\hline Enaba et al. (17) & 2015 & Cross-sectional & Egypt & $28.3 \pm 6.2$ & 35 & 2 \\
\hline Asadi et al. (18) & 2015 & Cross-sectional & Iran & $26.44 \pm 6.48$ & 69 & 15 \\
\hline Rizk et al. (13) & 2016 & Cross-sectional & Egypt & $29.7 \pm 6.7$ & 51 & 2 \\
\hline Ahmadimanesh et al. (19) & 2018 & Observational prospective & Iran & $22.8 \pm 5.8(14-37)$ & 47 & 3 \\
\hline Mohammadpour et al. (14) & 2009 & Cross-sectional & Iran & $25(14-35)$ & 43 & 17 \\
\hline
\end{tabular}

Table 3. Characteristics of studies on seizures due to tramadol use or abuse

\begin{tabular}{|c|c|c|c|c|c|c|}
\hline \multirow[t]{2}{*}{ Author } & \multirow{2}{*}{$\begin{array}{l}\text { Ingested dose of tramadol, } \\
\text { mean (min-max) }\end{array}$} & \multirow{2}{*}{$\begin{array}{l}\text { Route of } \\
\text { exposure }\end{array}$} & \multirow{2}{*}{$\begin{array}{l}\text { Type of } \\
\text { seizure }\end{array}$} & \multicolumn{2}{|c|}{ Manner of poisoning (\%) } & \multirow{2}{*}{$\begin{array}{l}\text { Number of seizure } \\
\text { episodes }\end{array}$} \\
\hline & & & & Prescribed & Abuse or suicide & \\
\hline Petramfar et al. (20) & $363.2 \pm 303.1(50-400)$ & Oral & Tonic-clonic & $18.9 \%$ & $81.1 \%$ & $\mathrm{~N}$ \\
\hline Talaie et al. (15) & $2059.35 \pm 226.04(500-4000)$ & Oral & Tonic-clonic & $9.7 \%$ & $65.5 \%$ & $\mathrm{~N}$ \\
\hline Goodarzi et al. (21) & $3248 \pm 2515(200-1100)$ & Oral & Tonic-clonic & $\mathrm{N}$ & $24 \%$ & $1-2(85 \%)$ \\
\hline Taghaddosinejad et al. (11) & $1511 \pm 1353(200-7000)$ & Oral & Tonic-clonic & $6.9 \%$ & $37 \%$ & $\mathrm{~N}$ \\
\hline Farajidana et al. (9) & $1416 \pm 1124(100-6000)$ & Oral & Tonic-clonic & $2.2 \%$ & $97 \%$ & $1-2(98 \%)$ \\
\hline Rahimi et al. (16) & $1395.7 \pm 218.3(100-12000)$ & Oral & $\mathrm{N}$ & 0 & $100 \%$ & $\mathrm{~N}$ \\
\hline Enaba et al. (17) & $1800(250-6750)$ & Oral & Tonic-clonic & $\mathrm{N}$ & $\mathrm{N}$ & Recurrent (81\%) \\
\hline Asadi et al. (18) & $140.17 \pm 73.53(50-300 \pm)$ & Oral & $\mathrm{N}$ & $\mathrm{N}$ & $\mathrm{N}$ & $\mathrm{N}$ \\
\hline Rizk et al. (13) & $1726 \pm 1049.1$ & Oral & Tonic-clonic & 0 & $100 \%$ & $\mathrm{~N}$ \\
\hline Ahmadimanesh et al. (19) & $1350(200-8000)$ & Oral & $\mathrm{N}$ & $2 \%$ & $94 \%$ & $\mathrm{~N}$ \\
\hline Mohammadpour et al. (14) & $491.90 \pm 435.54(45-1850)$ & Oral & $\mathrm{N}$ & $\mathrm{N}$ & $36 \%$ & $\mathrm{~N}$ \\
\hline
\end{tabular}

mean of $169.5 \pm 131.2 \mathrm{mg}$. The lowest dosage of tramadol dose causing a seizure reported among included studies was $50 \mathrm{mg}$ $(18,20)$.

Sub-group meta-analysis for Iranian studies with an overall number of 727 patients resulted in a mean of $1,416.4 \pm 356.0$ mg (95\% confidence interval: 718.5-2,114.2) for tramadol dosage but still the heterogeneity was significant $(Q=6106.52, I 2=99.90$, $\mathrm{p}<0.001$ ) (Figure 3).

\section{Characteristics of Patients with Tramadol Induced Seizure}

Mean and SD of the age of patients were reported in eight studies and three studies did not report SD $(11,14,15)$. The pooled data for patients' age, which were drawn from eight studies with 665 patients, showed a mean of $25.85 \pm 0.86$ years old (Figure 4$)$. The average proportion of males among all Data from 11 studies with
907 patients indicated the male gender as the most frequent patients (83.37 \pm 12.68 percent of the patients).

In all included studies, the patients used tramadol in oral form. Nine studies (786 patients) reported that the manner of poisoning in $70.51 \pm 29.07$ percent of the patients was suicide or abuse; however, two studies indicated that 23.2 and 27.2 percent of the patients had a pervious history of suicide. Moreover, data from four studies with 457 patients showed that 50.02 \pm 7.17 percent of the patients had an abuse of tramadol.

\section{Discussion}

Although the majority of included studies in our review were of high quality, they were considerably lacking adequate information to meticulously describe the policies of the researcher 


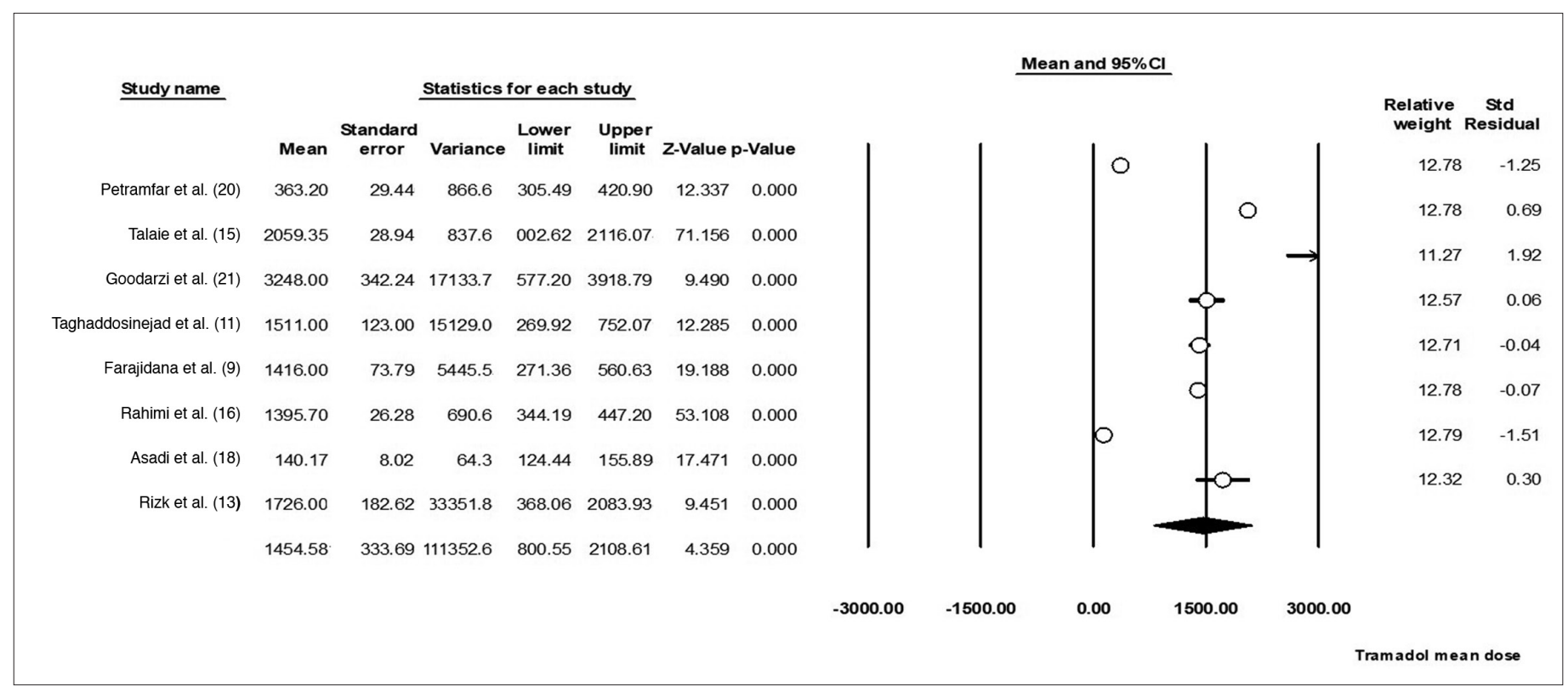

Figure 2. Meta-analysis of the dose of tramadol-induced seizure in all patients

$\mathrm{Cl}$ : Confidence interval, Std: Standard deviation

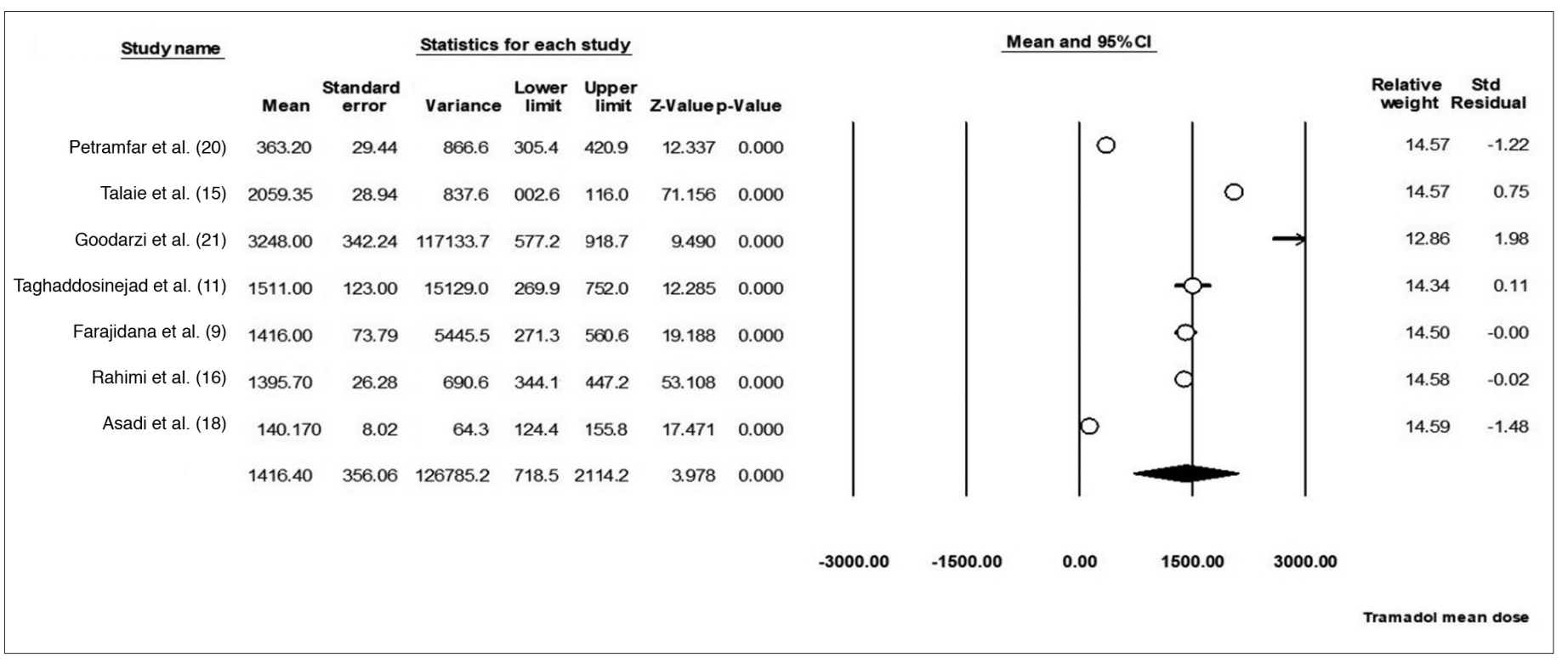

Figure 3. Meta-analysis of the dose of tramadol-induced seizure in the patients in Iran

Cl: Confidence interval, Std: Standard deviation

to ascertain a true seizure and consumption of tramadol. Also, the discrepancy between the data of included studies, especially for tramadol dosage, was considerable in majority of included studies $(9,11,13-16,19-21)$ and this discrepancy remained even after sub-group analysis of Iranian studies. Whereas ethnicity was the same, one of the possible reasons for this discrepancy could be inaccurate claims about the number of ingested tablets and their dosages due to the psychological stress of confronting a tonic-clonic seizure or forgetfulness. Three studies did not report the mean and SD or $p$ value of dose of ingested tramadol
$(14,18,19)$, therefore they were not included in the meta-analysis of the dose of ingested tramadol (Figures 2 and 3). Also, three studies did not report the mean and SD of age (only the median age was reported), therefore they were not included in the metaanalysis of age (Figure 4) $(11,14,15)$.

The included studies were mostly conducted in Iran and two of them were conducted in Egypt $(13,17)$; which both are developing countries. Therefore, it can be imagined that tramadol is conveniently available within these countries (20,23-25). 


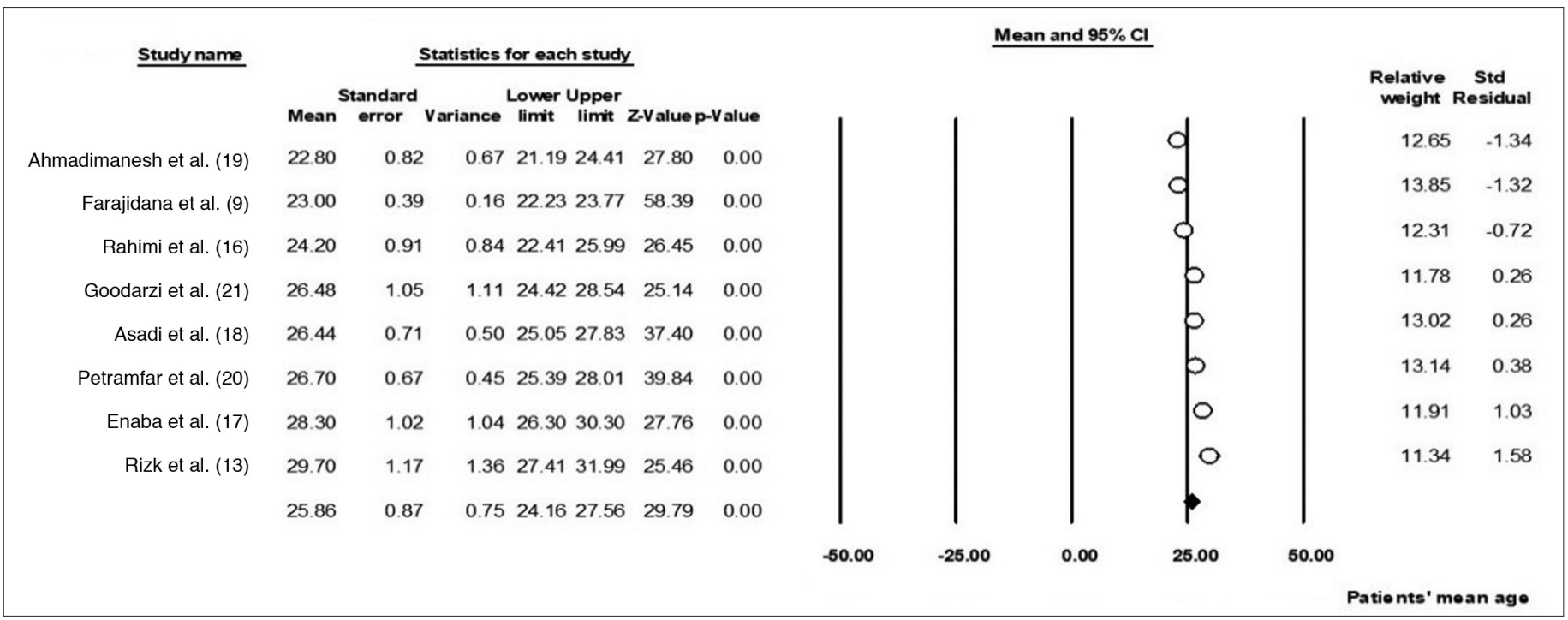

Figure 4. Meta-analysis of the age of patients with seizure due to tramadol

Cl: Confidence interval, Std: Standard deviation

A study used two definition of seizure including a broader and more specific definition and indicated that tramadol is not associated with a higher risk of seizure compared to codeine through a broader definition (based on either an outpatient physician claim for seizure disorder or a seizure-related emergency department visit or hospitalization); however, through using a more specific definition of seizure (restricted to a hospital visit with a definite diagnosis of seizure), they showed that tramadol is responsible for an increase of $41 \%$ in risk of seizure (26).

In almost all of the included studies, patients were in their early age of 20s and our estimations for their mean of age relieved the similar range. Moreover, in all of the included studies, male patients were more frequent than females. Although most of the patients admitted with tramadol induced seizure indicated that they used tramadol only for suicide or abuse, it is important to note that about one-third of the patients used this drug as a safe medication but still developed seizure. Moreover, about half the patients did not even have a history of tramadol abuse $(13,15$ 18,20). An Australian study indicated that tramadol overdose is associated with a significant risk of seizures in more severe cases, which appear to be related to the ingested dose; They found that seizures are apparently dose dependent and only occur in tramadol dosages of greater than $2000 \mathrm{mg}$, even in patients without a pre-existing risk of seizures (10).

Our study had some limitations including the considerable heterogeneity between the studies that prevented us to provide more reliable data. Also, this heterogeneity prevented us from drawing a definite conclusion based on the obtained data. Although we tried to reduce the heterogeneity by performing subgroup analysis, it was not that much effective. We included any studies conducted in both developing and developed countries. However, the studies conducted in developed countries were excluded because of our mentioned exclusion criteria. For example, the study of Ryan and Isbister (10) was excluded because they did not exclude the patients receiving co-ingestants or having seizure-related comorbidities when they calculated the mean dose of tramadol which induced seizure. We believe that these co-ingestants or seizure-related comorbidities may affect the dose of tramadol that causes seizure. Thus, we excluded these kinds of studies. Although our study failed to include the reports of the characteristics of patients in developed countries, there is no evidence in literature representing that there could be differences between characteristics of patients in developed and developing countries in terms of susceptibility to seizure due to tramadol. Therefore, this issue could not affect the generalizability of our results. In addition, our review only included the published studies, but we tried our best to contact with researchers and obtain the original data.

\section{Conclusion}

The current systematic review and meta-analysis study on tramadol induced seizure indicated that there was a substantial heterogeneity among studies on characteristics of patients with seizure due to tramadol. However, despite the extensive limitations of this study, our results demonstrated the higher prevalence of seizure due to tramadol in young men in developing countries, who mostly used tramadol in a suicide attempt or abuse. Moreover, due to possibility of seizure in patients with prescribed doses of tramadol, this complication should be considered by physicians. 


\section{Expert Opinion}

Previously tramadol was known as a cheap and safe drug with a low risk of addiction in comparison with other opiates (27), afterwards numerous studies reported severe adverse effects for it, such as seizure, rising the creatinine phosphokinase, acute renal failure, hepatic failure, electrocardiographic changes and acute right heart dysfunction and even death. The current study was mainly designed to review the published works on tramadol induced seizure and investigate the patients' demographics, previous history of drug use and seizure consumed tramadol dosage, and the manner of poisoning with tramadol.

Regarding the patients' demographics with tramadol induced seizure, the ones living in developing countries were the most frequent group of patients with tramadol induced seizure. Low awareness of tramadol side effects among people is another reason for high prevalence of tramadol poisoning and its consequences in developing countries such as Iran and Egypt $(13,26)$. According to an Egyptian study, the increasing violence forms are strictly associated with drug misuse. Low price of tramadol and easy access to it, compared to other abused drugs, and the unplanned and indirect advertisement of social media for tramadol could be other potential risk factors of tramadol poisoning $(27,28)$. Also, our findings indicated that individuals in male sex (83.37 \pm 12.6 percent) had higher prevalence of seizure following the consumption of tramadol. The social context, especially in developing countries, provides an easier access of tramadol for young men than the women (26,29-30), consequently, the prevalence of seizure due to tramadol is higher among the male users. Besides, young men have a higher tendency to do risky behaviors than women, so they are more prone to abusing drugs such as tramadol (30).

Our estimations for tramadol dosage inducing seizure in the patients, revealed a mean of $1,454.5 \pm 333.6 \mathrm{mg}$. Also, the minimum dose of tramadol causing seizure was estimated as $169.5 \pm 131.2 \mathrm{mg}$. Thus, if tramadol is being prescribed for therapeutic purposes, the physicians should properly make the patients aware about tramadol's complications; And if there is a high possibility of tramadol abuse in certain environments, more proper prevention programs using media and educational systems, especially in high schools and universities, are required to get administrated by governments against tramadol abuse targeting young population. Therefore, further studies are required to evaluate and introduce the most cost-benefit prevention programs against tramadol abuse, especially in context of developing countries.

\section{Ethics}

Peer-review: Externally peer-reviewed.

\section{Authorship Contributions}

Surgical and Medical Practices: S.S.V., Concept: P.H., Design: P.H., A.G., Data Collection or Processing: S.R.S.E., Analysis or Interpretation: R.Z., Literature Search: S.S.V., Writing: N.P., R.Z.

Conflict of Interest: No conflict of interest was declared by the authors.

Financial Disclosure: The authors declared that this study received no financial support.

\section{References}

1. Abdel-Hamid IA, Andersson KE, Waldinger MD, Anis TH. Tramadol Abuse and Sexual Function. Sexual medicine reviews. 2016;4:235-46.

2. Al-Abri SA, Woodburn C, Olson KR, Kearney TA. Ventricular Dysrhythmias Associated with Poisoning and Drug Overdose: A 10-Year Review of Statewide Poison Control Center Data from California. Am J Cardiovasc Drug. 2015; $15: 43-50$

3. Duke AN, Bigelow GE, Lanier RK, Strain EC. Discriminative stimulus effects of Tramadol in humans. Journal of Pharmacology and Experimental Therapeutics. 2011;338:255-62. [Epub 2011 Apr 5].

4. Knisely JS, Campbell ED, Dawson KS, Schnoll SH. Tramadol post-marketing surveillance in health care professionals. Drug and Alcohol Depend. 2002;68:15-22

5. The Statistical Centre of Iran. Iranian Population and Housing Census. Tehran, Iran: The Statistical Centre of Iran. 2016. https://www.unescap.org/ sites/default/files/Session6_Iran_Population_and_Housing_Census2016 Census_WS_24-26Jan2018.pdf

6. Tashakori A, Afshari R. Tramadol overdose as a cause of serotonin syndrome: a case series. Clinical Toxicology. 2010;48:337-41.

7. Moore KA, Cina SJ, Jones R, Selby DM, Levine B, Smith ML. Tissue distribution of Tramadol and metabolites in an overdose fatality. Am J Forensic Med Pathol. 1999;20:98-100.

8. Bekjarovski N, Chaparoska D, Radulovikj-Bekjarovska S. Seizures after use and abuse of Tramadol. Contributions (Prilozi). 2012;33:313-8.

9. Farajidana $H$, Hassanian-Moghaddam $H$, Zamani $N$, Sanai-Zadeh $H$. Tramadol-induced seizures and trauma. European Review for Medical \& Pharmacological Sciences. 2012;16(Suppl 1):34-7.

10. Ryan NM, Isbister GK. Tramadol overdose causes seizures and respiratory depression but serotonin toxicity appears unlikely. Clin Toxicol. 2015;53:54550

11. Taghaddosinejad F, Mehrpour O, Afshari R, Seghatoleslami A, Abdollahi M, Dart RC. Factors Related to Seizure in Tramadol Poisoning and Its Blood Concentration. J Med Toxicol. 2011;7:183-8.

12. Boostani R, Derakhshan S. Tramadol induced seizure: A 3-year study. Caspian Inton Med. 2012;3:484-7.

13. Rizk MMM, Kamal AM, MA Abdelhameed, Abdefadeel N, Hassan E, Khafagi ATM. Frequency and factors associated with occurrence of seizures in patients with Tramadol abuse: An upper egyptian experience. Addict Disord Treat. 2016;15:42-8.

14. Mohammadpour A, Ashkezari MD, Farahmand B, Shokrzadeh M. Demographic Characteristics and Functional Performance of the Kidneys and Hearts of Patients with Acute Tramadol Toxicity. Drug Research. 2019;69:20710.

15. Talaie H, Panahandeh R, Fayaznouri MR, Asadi Z, Abdollahi M. Doseindependent occurrence of seizure with Tramadol. J Med Toxicol. 2009;5:63-7.

16. Rahimi HR, Soltaninejad K, Shadnia S. Acute Tramadol poisoning and its clinical and laboratory findings. J Res Med Sci. 2014;19:855-9. 
17. Enaba D, Shalaby NM, El-Baz H, Zahra A. The Influence of A118G Single Nucleotide Polymorphism of Human Mu Opioid Receptor Gene and the MDR1 Gene in Egyptian Patients with Tramadol-induced Seizure. Addict Disord Treat. 2015;14:105-12.

18. Asadi P, Kasmaei VM, Ziabari SZ, Zohrevandi B, Manesh AM. Prevalence of Tramadol consumption in first seizure patients; a one-year cross-sectional study. Emerg. 2015;3:159-61.

19. Ahmadimanesh M, Shadnia S, Rouini MR, Sheikholeslami B, Ahsani Nasab S, Ghazi-Khansari M. Correlation between plasma concentrations of Tramadol and its metabolites and the incidence of seizure in Tramadol-intoxicated patients. DMPT. 2018;33:75-83.

20. Petramfar P, Borhani Haghighi A. Tramadol induced seizure: Report of 106 patients. Iranian Red Crescent Med J. 2010;12:49-51.

21. Goodarzi F, Mehrpour 0, Eizadi-Mood N. A study to evaluate factors associated with seizure in Tramadol poisoning in Iran. Indian J Forensic Med Toxicol. 2011;5:66-9.

22. Wells G, Shea B, O'connell D, Peterson J, Welch V, Losos M, et al. The Newcastle-Ottawa Scale (NOS) for assessing the quality of nonrandomized studies in meta-analyses. Department of Epidemiology and Community Medicine, University of Ottawa, Canada. University of Ottawa, Canada. 2012. http://www.ohri.ca/programs/clinical_epidemiology/oxford.asp

23. Afshari R, Afshar R, Mégarbane B. Tramadol overdose: review of the literature. Réanimation. 2011;20:436.
24. Shadnia S, Soltaninejad K, Heydari K, Sasanian G, Abdollahi M. Tramadol intoxication: a review of 114 cases. J Hum Exp Toxicol. 2008;27:201-5.

25. Gardner JS, Blough D, Drinkard CR, Shatin D, Anderson G, Graham D, et al. Tramadol and seizures: a surveillance study in a managed care population. Journal of Pharmacotherapy: J Hum Pharmacol. 2000;20:1423-31.

26. Morrow RL, Dormuth CR, Paterson M, Mamdani MM, Gomes T, Juurlink DN. Tramadol and the risk of seizure: nested case-control study of US patients with employer-sponsored health benefits. BMJ Open. 2019;9:e026705.

27. Sansone RA, Sansone LA. Tramadol: seizures, serotonin syndrome, and coadministered antidepressants. J Psychiat. 2009;6:17.

28. Fawzi M. Medicolegal aspects concerning Tramadol abuse. the new Middle East youth plague: an Egyptian overview 2010. Journal of forensic research. 2011, https://www.hilarispublisher.com/open-access/medicolegal-aspectsconcerning-tramadol-abuse-the-new-middle-east-youth-plague-anegyptian-overview-2010-2157-7145.1000130.pdf

29. Progler Y. Drug addiction in Gaza and the illicit trafficking of Tramadol. Journal of research in medical sciences: J Res Med Sci. 2010;15:185-8.

30. Cotto JH, Davis E, Dowling GJ, Elcano JC, Staton AB, Weiss SRB. Gender effects on drug use, abuse, and dependence: a special analysis of results from the National Survey on Drug Use and Health. Gender Med. 2010;7:402-13. 